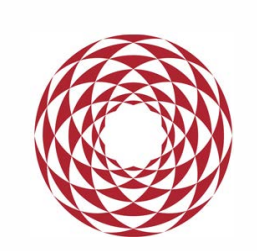

\title{
asHoka
}

UNIVERSITY

ASHOKA UNIVERSITY ECONOMICS

DISCUSSION PAPER NO. 47

\section{Strategy-Proof Club Formation with Indivisible Club Facilities}

December 2020

Bhaskar Dutta, Ashoka University

Anirban Kar, Delhi School of Economics

John A. Weymark, Vanderbilt University

https://ashoka.edu.in/economics-discussionpapers 


\title{
Strategy-Proof Club Formation with Indivisible Club Facilities
}

\author{
Bhaskar Dutta, Anirban Kar, and John A. Weymark
}

November 2020

\begin{abstract}
We investigate the strategy-proof provision and financing of indivisible club good facilities when individuals are subject to congestion costs that are nondecreasing in the number of other club members and in a private type parameter. An allocation rule specifies how the individuals are to be partitioned into clubs and how the costs of the facilities are to be shared by club members as a function of the types. We show that no allocation rule is strategy-proof and cost efficient (i.e., it always minimizes the aggregate of the financial and congestion costs of the club facilities) when congestion costs are strictly increasing in the type parameter, but that these properties are compatible if congestion costs are dichotomous and costs are equally shared within a club. We also provide examples of strategy-proof allocation rules with equal cost sharing that are (i) Pareto optimal and (ii) Pareto optimal, nondictatorial, and individually rational when the congestion cost is linear in the type parameter.
\end{abstract}

Key words: club goods; strategy-proofness

JEL classification number D71

\section{Introduction}

The degree of publicness of a good lies on a spectrum of possibilities ranging from the purely private to the purely public. In his theory of clubs, Buchanan (1965) addressed the problem of determining the optimal number of individuals with whom

Bhaskar Dutta

Department of Economics, University of Warwick, Coventry, CV4 7AL, UK and Department of Economics, Ashoka University, Plot No. 2, Rajiv Gandhi Education City, P. O. Rai, Sonepat, Haryana 131029, India

e-mail: bhaskar.dutta@ashoka.edu.in

Anirban Kar

Department of Economics, Delhi School of Economics, University of Delhi, Delhi 110007, India

e-mail: anirban@econdse.org

John A. Weymark

Department of Economics, Vanderbilt University, PMB 351819, 2301 Vanderbilt Place, Nashville, TN 37235-1819, USA.

e-mail: john.weymark@vanderbilt.edu 
to share the consumption of a good when the benefits and costs of belonging to a sharing group - a club - depend on both the amount of the good and the number of individuals the club good is shared with. Buchanan was concerned with the problem of determining the optimal size of a single club. In contrast, Tiebout (1956) was interested in investigating the sorting of individuals into communities through locational choices so as to consume local public goods, which are goods that are non-rival in consumption within a community but provide no benefits to outsiders. Communities in Tiebout's model can be thought of as being clubs in Buchanan's sense. ${ }^{1}$

In this article, we investigate the partitioning of a fixed group of individuals into clubs so as to share the benefits and costs of a single club good from which nonmembers can be excluded. We assume that each club is self-financing, so there is no cross-subsidization across clubs. The benefits of a club good are public to the members of a club, but are subject to congestion costs due to the negative externality that arises when a club good is shared with other people. Examples include community swimming pools and parks. As in these examples, we regard a club good as being some form of infrastructure, what we henceforth call a facility. We consider the case in which each facility is indivisible and is produced with a common fixed cost. The congestion cost experienced by an individual depends on both the number of individuals in his club and on his own characteristics. The latter is his type, which is private information.

An allocation rule specifies how the individuals are to be partitioned into clubs and how the costs of the facilities are to be shared by club members as a function of the types of the individuals. We are interested in determining which allocation rules, if any, are strategy-proof and satisfy one or more additional desirable properties when the congestion cost is non-decreasing in both the number of individuals a club is shared with and the value of the type parameter. Strategy-Proofness is the requirement that everybody always has an incentive to report his true type. By assuming that the size of a facility is fixed, we are able to focus on how the design of allocation rules that satisfy the properties that we consider depend on congestion effects in isolation from any facility size considerations.

In addition to Strategy-Proofness, we consider four other desirable properties for an allocation rule: Cost Efficiency, Pareto Optimality, Nondictatorship, and Individual Rationality. Cost Efficiency requires the partition of individuals into clubs to minimize the sum of the total financial cost of the club facilities and the aggregate congestion cost. Pareto Optimality requires that allocations be strictly Pareto optimal. Nondictatorship requires that nobody always has one of his most preferred allocations chosen. Individual Rationality requires that nobody is ever worse off being assigned to a multi-member club than being in his own single-member club.

We prove that no allocation rule can satisfy Strategy-Proofness and Cost Efficiency if the congestion cost function is strictly increasing in the type parameter. However, if congestion costs can only take on two values, we show that a serial dictatorship satisfies both of these properties if each club's financial cost is shared

${ }^{1}$ An introduction to the literature on club goods and local public goods may be found in Cornes and Sandler (1996). 
equally among its members. When investigating the compatibility of StrategyProofness with our other three axioms (i.e., excluding Cost Efficiency), we restrict attention to the case in which members share the cost of a club facility equally and congestion costs are linear in the type parameter. We show by means of an example that these four properties can be satisfied simultaneously. Because an individual only cares about the number of people he shares a club with, and not who they are, to ensure that strictly Pareto optimal allocations are chosen by a strategy-proof allocation rule, individual indifferences between club partitions must be properly taken account of. We illustrate this observation by considering two ways of specifying a serial dictatorship, only one of which always chooses a strictly Pareto optimal allocation. $^{2}$

In Section 2, we discuss some related literature. We present the model in Section 3 and the axioms in Section 4. The compatibility of Strategy-Proofness and Cost Efficiency is considered in Section 5. Section 6 provides an example that demonstrates the compatibility of Strategy-Proofness with the other axioms when the are three individuals. In order to illustrate the difficulty of satisfying all of these axioms, we consider some further examples in Section 7. Finally, in Section 8, we offer some concluding remarks.

\section{Related Literature}

While there is an extensive literature on club formation, little attention has been devoted to this issue when individuals have private information about the benefits and costs of a club. As a consequence, little is known about the incentive issues involved when determining club membership. To the best of our knowledge, there are only three other papers that examine the strategy-proof provision of club goods. Jackson and Nicolò (2004) and Long (2019) have considered the problem of determining the membership of a single club, while Bogomolnaia and Nicolò (2005) have considered the problem of partitioning the individuals into a fixed number of clubs. For Long, the club good has a fixed size, whereas for Jackson and Nicolò and Bogomolnaia and Nicolò, the location of the club good in $[0,1]$ (which can be interpreted as the quantity of the good) must also be determined. In each of these articles, the private information is about the preferences of the individuals. Like Bogomolnaia and Nicolò, we are concerned with the problem of partitioning the individuals into clubs, but we allow the number of clubs to be endogenously determined. Like Long, we assume that the club good is of fixed size.

Jackson and Nicolò (2004) assumed that an alternative is characterized by two attributes-the location of a club good facility in $[0,1]$ and the number of individuals sharing it. An individual only cares about a facility's location if he belongs to the club. Preferences over possible locations are single-peaked, with the peak being independent of the club membership. An individual also has preferences over the size

\footnotetext{
${ }^{2}$ A serial dictatorship is strategy-proof and always chooses a weakly Pareto optimal allocation.
} 
of the club (but not its composition) if he is a member and the option of not being in the club, with preferences over group size assumed to be single-peaked. Jackson and Nicolò considered four alternative restrictions on the club size preferences in addition to the requirement that they be single-peaked. In three of them, everybody agrees on the most preferred club size: (i) a single-member club (the congestion effect dominates), (ii) a club with everybody in it (the cost-sharing effect dominates), or (iii) some intermediate-sized club. In their fourth preference domain, there are two possible most-preferred club sizes. They have established a number of possibility and impossibility results for a strategy-proof allocation rule when various other desirable properties are required. The nature of the results depends on which of the four preference domains is considered. Of particular note is their finding that strategy-proofness and Pareto optimality imply that the number of club members must be independent of the locational preferences. When the location of the club good is required to depend only on the preferences of the individuals who are members of the club, then the club has only one member in case (i) and consists of the whole group in case (ii). In the former case, this person-a dictator-chooses the facility location; in the latter case, it is chosen using a generalized median rule. In case (iii), the location must coincide with the peak of one of the club members, and so any member whose locational peak diverges sufficiently from this value might prefer not to be in the club, which is inconsistent with voluntary participation.

Bogomolnaia and Nicolò (2005) also assumed that an alternative is characterized by the location of a club good facility in $[0,1]$ and the number of individuals sharing it. Their allocation rule specifies the locations of a fixed number of club good facilities and partitions the individuals among them. Unlike Jackson and Nicolò (2004), there is no option of not joining a club. Individual preferences over locations are single-peaked. Conditional on club size, being closer to the locational peak is preferred, and conditional on the facility location, having a smaller club is preferred. When there are only two club good facilities, Bogomolnaia and Nicolò have shown that it is possible to find allocation rules that are strategy-proof, Pareto optimal, and satisfy a stability property that requires no individual to want to change the club he is assigned to. However, with more than two club facilities, these conditions are incompatible.

Long (2019) considered the problem of selecting which individuals are to form a club out of a group of $n$ individuals. Each individual has strict, single-peaked preferences over being in a club with $k \in\{0,1, \ldots, n\}$ members, where 0 is the option of not joining any club. Long focused on the construction of strategy-proof rules satisfying Pareto optimality and a voluntary participation constraint that ensures that no one is forced to join the club if that is worse than not joining. ${ }^{3}$ She identified two interesting classes of rules satisfying these properties, both of which satisfy some form of group strategy-proofness. One involves individuals who wish to do so joining a club in a fixed priority order until someone who has already joined objects. The other involves individuals voting on club group size in ascending order subject to a stopping rule. In Section 7, we define an iterative version of this procedure that re-

\footnotetext{
${ }^{3}$ Not joining any club is not an option in our model.
} 
sults in a partition of the individuals into clubs and show that it is not strategy-proof when costs are shared equally.

Strategy-proofness is not the only way to model individual incentives in the presence of private information. For the problem of determining which of a finite set of possible levels of a single club good to provide and who is to share it, Massó and Nicolò (2008) have studied which collective choice rules are Nash or Subgame Perfect Nash implementable when individuals prefer larger groups in the sense of set inclusion. They have shown that Subgame Perfect Nash implementability is compatible with Pareto optimality and a stability property that requires that nobody can be excluded from access to the good or forced to consume it against his wishes, whereas Nash implementability is not.

Finally, we note that our problem of partitioning individuals into clubs contributes to the more general literature on coalition formation. ${ }^{4}$ As we have already mentioned, communities in the Tiebout (1956) problem can be thought of as being clubs that are not subject to congestion effects. With models of hedonic coalition formation, an individual's preferences only depend on the composition of the coalition that he belongs to. See, for example, Bogomolnaia and Jackson (2002). Clubs are coalitions and its members' preferences only depend on group size rather than on their identities, and so are non-hedonic. The model of group activity selection studied by Darmann (2019) in which individuals are to be assigned to at most one of a set of possible activities is also closely related. In Darmann's model, individuals have preferences over pairs consisting of an activity and the number of individuals participating in it. In our model, individuals do not care about which club they belong to, only how many members it has.

\section{The Model}

There is a group $N=\{1, \ldots, n\}$ consisting of $n \geq 2$ individuals. Individuals form clubs (subgroups of $N$ ) so as to self-finance indivisible club good facilities whose benefits are public to the members of a club but from which outsiders are excluded. There is a fixed cost of producing a facility, which we normalize to equal 1. Individuals in a club do not care about the identities of its members, only their number and how the costs of their facility is shared.

Let $\mathscr{P}$ denote the set of all partitions of $N$, with typical element $P$. Given $P \in \mathscr{P}$, $S_{i}(P)$ is the element of $P$ that contains $i ; S_{i}(P)$ is $i$ 's club. The number of other individuals who are in $i$ 's club is $n_{i}$, where $n_{i} \in N^{\circ}=\{0, \ldots, n-1\}$. We write $n_{i}\left(S_{i}(P)\right)$ when we want to explicitly note the dependence of $n_{i}$ on which club in the partition $P$ that $i$ belongs to. Thus, $S_{i}(P)$ contains $n_{i}\left(S_{i}(P)\right)+1$ individuals.

Club good facilities are congestible. The congestion cost experienced by individual $i$ depends on both the number of individuals in $i$ 's club and on $i$ 's own characteristics, which we assume can be summarized by a scalar $\alpha_{i}$. This is $i$ 's type. It can

\footnotetext{
${ }^{4}$ For a survey, see Bloch and Dutta (2011).
} 
take on any non-negative value. The value of $\alpha_{i}$ is private information to individual $i$. We let $\alpha=\left(\alpha_{1}, \ldots, \alpha_{n}\right)$ denote a vector of types.

Individual $i$ 's congestion cost $i$ is $f\left(\alpha_{i}, n_{i}\right)$, where $f\left(\alpha_{i}, \cdot\right)$ is non-decreasing in $n_{i}$ and $f\left(\cdot, n_{i}\right)$ is non-decreasing in $\alpha_{i}$. In other words, the congestion cost is nondecreasing in both club size and an individual's type. There is no congestion cost if a club only has one member, so $f\left(\alpha_{i}, 0\right)=0$ for all types. Note that it is assumed that the function $f$ is the same for all individuals; all person-specific congestion effects are captured by the type parameters.

Congestion costs are measured in monetary units, so $f\left(\alpha_{i}, n_{i}\right)$ can be interpreted as being the amount by which an individual of type $\alpha_{i}$ who shares a facility with $n_{i}$ other individuals must be compensated in order to be indifferent to being the only person in the club. With this interpretation of congestion costs, the function $f$ represents an individual's preferences for sharing a facility. Measuring congestion costs in monetary units permits us to compare these costs with the monetary cost of providing a facility.

We further assume that (i) there is a value of the type parameter $a^{*}$ such that $f\left(a^{*}, 1\right)>1$ and (ii) $f\left(0, n_{i}\right)=0$ for all $n_{i} \in N^{\circ}$. The first assumption requires the congestion cost to be larger than the financial cost of producing the facility for sufficiently high values of the type parameter even if there is only one other club member. The second assumption simply says that if an individual does not care about how many people the facility is shared with (i.e., his type is 0 ), then there is no congestion cost.

Two kinds of congestion cost functions that are of particular interest here are those with linear congestion costs and those with dichotomous costs.

Linear Congestion Costs. For all $i \in N$, all $\alpha_{i} \in \mathbb{R}_{+}$, and all $n_{i} \in N^{\circ}, f\left(\alpha_{i}, n_{i}\right)=$ $\alpha_{i} n_{i}$.

With linear congestion costs, the congestion cost experienced by any individual is proportional to the number of other people the facility is shared with, with the factor of proportionality given by his type.

Dichotomous Congestion Costs. For all $i \in N$, all $\alpha_{i} \in \mathbb{R}_{+}$, and all $n_{i} \in N^{\circ}, f\left(\alpha_{i}, n_{i}\right)=$ $c>1$ if $n_{i}>\alpha_{i}$ and $f\left(\alpha_{i}, n_{i}\right)=0$ otherwise.

With dichotomous congestion costs, $i$ 's type $\alpha_{i}$ specifies a threshold. If the number of other members of his club falls below this threshold, then he does not experience any congestion cost. However, if the threshold is met, then the congestion cost is some fixed value $c$ that exceeds the cost of the facility regardless of by how much $\alpha_{i}$ exceeds the threshold. In keeping with our assumption that $\alpha_{i}$ can be any nonnegative number, the threshold need not be an integer. This is of no consequence because the value of $f$ is constant in $\alpha_{i}$ for all $\alpha_{i}$ in an interval of the form $[j, j+1)$, where $j$ is a non-negative integer.

We assume that each club is responsible for fully financing its own facility, so there is no cross-subsidization of a facility's cost between clubs. An allocation $(P, r)=\left(P, r_{1}, \ldots, r_{n}\right)$ consists of a partition $P$ of the individuals into clubs and the 
amount $r_{i} \geq 0$ that each individual $i \in N$ contributes to the cost of the facilities, with $\sum_{i \in S} r_{i}=1$ for all $S \in P$. An allocation rule specifies an allocation as a function of the individuals' types. Formally, an allocation rule is a function $A: \mathbb{R}_{+}^{n} \rightarrow \mathscr{P} \times \mathbb{R}_{+}^{n}$, where for all $\left.\alpha \in \mathbb{R}_{+}^{n}, A(\alpha)=(P(\alpha), r(\alpha))=\left(P(\alpha), r_{1}(\alpha)\right), \ldots, r_{n}(\alpha)\right)$. In this definition, $P(\cdot)$ is the partition chosen and $r_{i}(\cdot)$ is how much $i$ must pay towards the cost of $i$ 's club good facility as a function of the type vector.

An allocation rule has equal cost sharing if the cost of a facility is always shared equally among the club members.

Equal Cost Sharing. An allocation rule $A$ has equal cost sharing if for all $\alpha \in \mathbb{R}_{+}^{n}$ and all $i \in N, r_{i}(\alpha)=1 /\left|S_{i}(P(\alpha))\right|$.

Given $\alpha$, the aggregate cost associated with a partition $P \in \mathscr{P}$ is given by

$$
C(P, \alpha)=|P|+\sum_{i \in N} f\left(\alpha_{i}, n_{i}\left(S_{i}(P)\right)\right) .
$$

The first term is the financial cost of building the $|P|$ facilities associated with the partition $P$, while the second term is the aggregate congestion cost associated with it.

Each individual wants to minimize the sum of the amount paid towards the cost of the club facility and the congestion cost associated with it. Thus, individual $i$ with type $\alpha_{i}$ and cost share $r_{i}$ who belongs to a club with $n_{i}$ other members has utility

$$
U\left(\alpha_{i}, r_{i}, n_{i}\right)=-\left[r_{i}+f\left(\alpha_{i}, n_{i}\right)\right] .
$$

A person's type captures all person-specific effects on utility, so the functional form of the utility function is not indexed by the names of the individuals. If there are linear congestion costs and the cost of a club good facility is shared equally among the club members, then

$$
U\left(\alpha_{i}, r_{i}, n_{i}\right)=-\left[\frac{1}{n_{i}}+\alpha_{i} n_{i}\right]
$$

\section{The Axioms}

We are interested in strategy-proof allocation rules. These are rules for which type misrepresentation is never advantageous.

Strategy-Proofness. An allocation rule $A$ is strategy-proof if for all $\alpha \in \mathbb{R}_{+}^{n}$, all $i \in N$, and all $\alpha_{i}^{\prime} \in \mathbb{R}_{+}$,

$$
U\left(\alpha_{i}, r_{i}, n_{i}\left(S_{i}(P)\right)\right) \geq U\left(\alpha_{i}, r_{i}^{\prime}, n_{i}\left(S_{i}\left(P^{\prime}\right)\right)\right),
$$

where $(P, r)=A(\alpha)$ and $\left(P^{\prime}, r^{\prime}\right)=A\left(\alpha_{-i}, \alpha_{i}^{\prime}\right)$. 
It is a simple matter to construct strategy-proof allocation rules. For example, an allocation rule with equal cost-sharing is strategy-proof if the choice of partition is independent of the type vector. This is clearly an unsatisfactory allocation rule. Hence, Strategy-Proofness must be supplemented with one or more additional properties to be of interest. We consider four such properties.

A partition $P \in \mathscr{P}$ is cost efficient at $\alpha$ if there is no other partition $P^{\prime} \in \mathscr{P}$ such that $C\left(P^{\prime}, \alpha\right)<C(P, \alpha)$. A cost efficient partition minimizes aggregate cost. Note that cost efficiency does not depend on how the facility costs are shared among the individuals.

Cost Efficiency. An allocation rule $A$ is cost efficient if for all $\alpha \in \mathbb{R}_{+}^{n}, P(\alpha)$ is cost efficient at $\alpha$.

An allocation $(P, r) \in \mathscr{P} \times \mathbb{R}_{+}^{n}$ is Pareto optimal at $\alpha$ if there is no other allocation $\left(P^{\prime}, r^{\prime}\right) \in \mathscr{P} \times \mathbb{R}_{+}^{n}$ such that for all $i \in N$,

$$
U\left(\alpha_{i}, r_{i}^{\prime}, n_{i}\left(S_{i}\left(P^{\prime}\right)\right)\right) \geq U\left(\alpha_{i}, r_{i}, n_{i}\left(S_{i}(P)\right)\right)
$$

and there exists an $i \in N$ such that

$$
U\left(\alpha_{i}, r_{i}^{\prime}, n_{i}\left(S_{i}\left(P^{\prime}\right)\right)\right)>U\left(\alpha_{i}, r_{i}, n_{i}\left(S_{i}(P)\right)\right) .
$$

In other words, an allocation is Pareto optimal if there is no other allocation that makes everybody as least as well off and one or more individuals strictly better off.

Pareto Optimality. An allocation rule $A$ is Pareto optimal if for all $\alpha \in \mathbb{R}_{+}^{n}, A(\alpha)$ is Pareto optimal at $\alpha$.

Individual $d \in N$ is a dictator for the allocation rule $A$ if for all $\alpha \in \mathbb{R}_{+}^{n}$ and any allocation $(P, r) \in \mathscr{P} \times \mathbb{R}_{+}^{n}$,

$$
U\left(\alpha_{d}, r_{d}(\alpha), n_{i}\left(S_{d}(P(\alpha))\right) \geq U\left(\alpha_{d}, r_{d}, n_{d}\left(S_{d}(P)\right)\right) .\right.
$$

That is, there is some individual $d$-the dictator-for whom for each type vector $\alpha$, the allocation rule $A$ selects a partition of the individuals into clubs and assigns facility cost shares so as to minimize the sum of $d$ 's congestion cost and $d$ 's cost share. A dictator never contributes to the cost of a club facility if someone other than the dictator is in his club.

Nondictatorship. An allocation rule $A$ is nondictatorial if there is no dictator.

An allocation $(P, r) \in \mathscr{P} \times \mathbb{R}_{+}^{n}$ is individually rational at $\alpha$ if for all $i \in N$,

$$
U\left(\alpha_{i}, r_{i}, n_{i}\left(S_{i}(P)\right)\right) \geq U\left(\alpha_{i}, 1,0\right) .
$$

With an individually rational allocation, nobody can be made worse off by being in a club with one or more other members instead of having his own single-member club. When this condition is satisfied, club membership is voluntary.

Individual Rationality. An allocation rule $A$ is individually rational if for all $\alpha \in$ $\mathbb{R}_{+}^{n}, A(\alpha)$ is individually rational at $\alpha$. 


\section{Strategy-Proofness and Cost Efficiency}

We begin our analysis by considering the compatiblity of Strategy-Proofness and Cost Efficiency. We have assumed that the congestion cost function $f$ is nondecreasing in the type parameter for a given number of club members. We show that if $f$ is strictly increasing in the type parameter, then it is not possible for an allocation function to be both strategy-proof and cost efficient.

Theorem 1. If $f\left(\cdot, n_{i}\right)$ is strictly increasing in its first argument for all $n_{i} \in N^{\circ}$, then there is no allocation rule that satisfies Strategy-Proofness and Cost Efficiency.

Proof. (i) We first consider the case in which $n=2$.

Let $R=\left\{\alpha \in \mathbb{R}_{+}^{2} \mid f\left(\alpha_{1}, 1\right)+f\left(\alpha_{2}, 1\right)<1\right\}$. Note that for all $\alpha \in R$, Cost Efficiency implies that the allocation rule must assign both individuals to the same club.

We begin by showing that for all $\alpha, \alpha^{\prime} \in R, r(\alpha)=r\left(\alpha^{\prime}\right)$. There are three cases to consider.

First, suppose that $\alpha_{i}>\alpha_{i}^{\prime}$ and $\alpha_{j}<\alpha_{j}^{\prime}$, where $i \in\{1,2\}$ and $i \neq j$. Let $\bar{\alpha}$ be such that $\bar{\alpha}_{i}=\alpha_{i}^{\prime}$ and $\bar{\alpha}_{j}=\alpha_{j}$. Note that $\bar{\alpha} \in R$, and so both individuals must be in the same club at $\bar{\alpha}$. If $r_{i}(\alpha)>r_{i}(\bar{\alpha})$, then $i$ can manipulate at $\alpha$ by reporting $\alpha_{i}^{\prime}$. Conversely, if $r_{i}(\alpha)<r_{i}(\bar{\alpha})$, then $i$ can manipulate at $\bar{\alpha}$ by reporting $\alpha_{i}$. Thus, by Strategy-Proofness, we must have $r(\alpha)=r(\bar{\alpha})$. For analogous reasons, we must have $r\left(\alpha^{\prime}\right)=r(\bar{\alpha})$. Hence, $r(\alpha)=r\left(\alpha^{\prime}\right)$.

Second, suppose that $\alpha_{i}>\alpha_{i}^{\prime}$ for $i=1,2$. Let $\bar{\alpha}$ be such that $\bar{\alpha}_{i}=\alpha_{i}$ and $\bar{\alpha}_{j}=\alpha_{j}^{\prime}$. Then, $r\left(\alpha^{\prime}\right)=r(\bar{\alpha})$, for otherwise $i$ can manipulate. Similarly, $r(\alpha)=r(\bar{\alpha})$, for otherwise $j$ can manipulate. Hence, $r(\alpha)=r\left(\alpha^{\prime}\right)$.

Third, suppose that $\alpha_{i} \geq \alpha_{i}^{\prime}$ for $i=1,2$ with just one strict inequality. The proof for this case is almost identical to the proof of the preceding case.

For $\alpha \in R$, we have shown that the individual cost shares are independent of the types. Because the cost shares are non-negative and sum to 1 , it then follows that for every $\alpha \in R$, either 1 pays at least $1 / 2$ or 2 pays at least $1 / 2$. Without loss of generality, suppose that 1 pays at least half the cost of the club. Now, choose $\hat{\alpha}_{1}$ such that $1 / 2<f\left(\hat{\alpha}_{1}, 1\right)<1$. Because $f\left(0, n_{i}\right)=0$ and there exists an $a^{*} \in \mathbb{R}_{+}$ such that $f\left(a^{*}, 1\right)>1$, the strict increasingness of $f$ in its first argument implies that such an $\hat{\alpha}$ exists. Let $\hat{\alpha}=\left(\hat{\alpha}_{1}, 0\right)$. By construction, $\hat{\alpha}$ is in $R$. We have that $U\left(\hat{\alpha}_{1}, r_{1}(\hat{\alpha}), n_{1}\left(S_{1}(P(\hat{\alpha}))\right)=U\left(\hat{\alpha}_{1}, r_{1}(\hat{\alpha}), 1\right)<-3 / 2\right.$.

If individual 1 announces $\alpha_{1}^{\prime}=a^{*}$, then $\alpha^{\prime}=\left(\alpha_{1}^{\prime}, 0\right) \notin R$ and the cost efficient partition puts 1 and 2 in separate clubs. With this partition, 1 does not suffer any congestion cost, but pays the full cost of a club good facility. Thus, $U\left(\alpha_{1}^{\prime}, r_{1}\left(\alpha^{\prime}\right), n_{1}\left(S_{1}\left(P\left(\alpha^{\prime}\right)\right)\right)\right)=U\left(\alpha_{1}^{\prime}, 1,0\right)=-1$. Hence, 1 can manipulate at $\alpha$ by reporting $\alpha_{1}^{\prime}$. Therefore, when $n=2$, it not possible to satisfy both StrategyProofness and Cost Efficiency.

(ii) We now consider the case in which $n>2$. Let $R^{\prime}=\left\{\alpha \in \mathbb{R}_{+}^{n} \mid f\left(\alpha_{i}, 1\right) \geq\right.$ 1 for all $i>2\}$. Then, for all $\alpha \in R^{\prime}$ such that $\left(\alpha_{1}, \alpha_{2}\right) \in R$, Cost Efficiency and the non-decreasingness of $f$ in its second argument imply that individuals 1 and 2 share 
a facility, while everybody else forms a singleton club. For such an $\alpha$, by applying the preceding argument for $n=2$, we conclude that Strategy-Proofness and Cost Efficiency are inconsistent with having a two-person club.

For some values of the type vector $\alpha$, it is optimal to have a two-person club. One of the members of this club, say $i$, must pay at least half of the cost of this club facility. The proof of Theorem 1 shows that by reporting a sufficiently large value of $\alpha_{i}$ (i.e., by claiming to be strongly negatively affected by the presence of other club members), Cost Efficiency requires $i$ to instead form his own single-member club, which is beneficial for him because his increased facility charge is more than offset by the absence of any congestion cost. But if that is the case, Strategy-Proofness is violated.

Further intuition for this result may be obtained by considering what $i$ considers when deciding what to report when his true type is $\alpha_{i}$. Suppose that $i$ misrepresents by, say, reporting $\alpha_{i}^{\prime}$. If this results in the same club partition, then the only difference in aggregate cost is due to the change in the sum of $i$ 's congestion and facility costs, which $i$ takes account of when deciding what type to report. However, if the membership of $i$ 's club differs when $\alpha_{i}^{\prime}$ is reported, then the aggregate cost also differs due to the change in the costs that the other individuals experience. The failure to take account of this externality underlies the incompatibility of StrategyProofness and Cost Efficiency, much like the failure to take account of a negative externality underpins the Tragedy of the Commons (Hardin, 1968).

In Theorem 1, no restrictions are placed on how the costs of the club facilities are shared except for the maintained assumptions that the payments are non-negative and that club members pay for their own facility themselves. If we further suppose that club members share the cost of their facility equally, the incompatibility of Strategy-Proofness and Cost Efficiency is preserved. Indeed, the preceding proof also establishes this impossibility result.

Corollary. If $f\left(\cdot, n_{i}\right)$ is strictly increasing in its first argument for all $n_{i} \in N^{\circ}$, then there is no allocation rule with equal cost sharing that satisfies Strategy-Proofness and Cost Efficiency.

If there are dichotomous congestion costs, then the congestion cost function $f$ is weakly, but not strictly, increasing in the type parameter, and so the preceding results do not apply. ${ }^{5}$ When congestion costs are dichotomous, it is possible to find allocation rules that are both strategy-proof and cost efficient. We show that this is so in Theorem 2 for the case in which there is equal cost sharing.

Theorem 2. If there are dichotomous congestion costs, then there is an allocation rule satisfying Strategy-Proofness, Cost Efficiency, Individual Rationality, and Nondictatorship.

\footnotetext{
${ }^{5}$ In the proof of Theorem 1, strict increasingness (rather than weak increasingness) of $f$ in the type parameter is only used to show the existence of an $\hat{\alpha}_{1} \in\left(0, a^{*}\right)$ for which $1 / 2<f\left(\hat{\alpha}_{1}, 1\right)<1$. This is not possible with dichotomous congestion costs.
} 
Proof. First, we remark that in the presence of dichotomous costs, Cost efficiency implies Individual Rationality. To see this, recall that with dichotomous congestion costs, $i$ 's type $\alpha_{i}$ serves a threshold. If the number of other club members exceeds this threshold, then $f\left(\alpha_{i}, n_{i}\right)=c>1$; otherwise $f\left(\alpha_{i}, n_{i}\right)=0$. By Cost Efficiency, when the type vector is $\alpha, i$ must be assigned to a club with no more than $\left\lfloor\alpha_{i}+1\right\rfloor$ club members, where $\lfloor a\rfloor$ denotes the largest integer that does not exceed $a$. To see why, suppose on the contrary that $\left|S_{i}(P(\alpha))\right|>\left\lfloor\alpha_{i}+1\right\rfloor$. By partitioning $S_{i}(P(\alpha))$ into two clubs, one of which only includes $i$, the total financial cost of the facilities increases by 1 . Individual $i$ 's congestion cost decreases by $c>1$. The congestion costs of the other original members of $S_{i}(P(\alpha))$ either do not change or are reduced by $c$. Nobody else's congestion cost changes. Thus, the sum of the congestions costs declines more than the increase in the financial cost of the facilities, contradicting the cost efficiency of $P(\alpha)$. Thus, if a partition is cost efficient, there are no congestion costs, and so Individual Rationality is satisfied.

We construct an allocation rule that has equal cost sharing. Let $\succ$ be a linear order of the partitions in $\mathscr{P}$, with $P \succ P^{\prime}$ interpreted as meaning that $P$ precedes $P^{\prime}$ in this order. For any type vector $\alpha$, let $\mathscr{E}(\alpha)$ denote the set of cost efficient partitions. The allocation rule is a serial dictatorship using the natural order of the individuals, $1, \ldots, n$, in which the partition choices are restricted to the cost efficient partitions. More precisely, for all $\alpha \in \mathbb{R}_{+}^{n}$, the partition $P(\alpha)$ is determined sequentially as follows. First, individual 1 chooses his utility maximizing partitions $\mathscr{E}_{1}(\alpha)$ in $\mathscr{E}(\alpha)$. Second, individual 2 chooses his utility maximizing partitions $\mathscr{E}_{2}(\alpha)$ in $\mathscr{E}_{1}(\alpha)$. Each person gets to choose a set of cost efficient partitions in turn until the set $\mathscr{E}_{n}(\alpha)$ is identified. $P(\alpha)$ is the first partition in this set according to $\succ$. Note that if a unique partition is identified at any stage in this process, then it is $P(\alpha)$. By construction, the allocation rule that chooses partitions in this way and shares costs equally among club members satisfies Cost Efficiency.

The allocation rule satisfies Non-dictatorship. For suppose there is $i$ for whom $\alpha_{i}>n-1$. Then, $i$ prefers to be in a club with all other individuals. However, this may not be cost efficient since some other individual $j$ may have $\alpha_{j}<n-1$.

We now show that it also satisfies Strategy-Proofness.

Consider any type $\alpha \in \mathbb{R}_{+}^{n}$ and any individual $i \in N$. To show that the allocation rule is strategy-proof, we need to show that any reported type $\alpha_{i}^{\prime}$ different from $i$ 's true type $\alpha_{i}$ does not make him better off. Let $\alpha^{\prime}=\left(\alpha_{1}, \ldots, \alpha_{i-1}, \alpha_{i}^{\prime}, \alpha_{i+1}, \ldots, \alpha_{n}\right)$. There are two cases: (i) $\alpha_{i}^{\prime}<\alpha_{i}$ and (ii) $\alpha_{i}^{\prime}>\alpha_{i}$.

(i) Suppose that $\alpha_{i}^{\prime}<\alpha_{i}$. If $\alpha_{i}^{\prime}<n_{i}\left(S_{i}(P(\alpha))\right)$, then $P(\alpha)$ is not cost efficient for $\alpha^{\prime}$ because Cost Efficiency requires $i$ to be in a club with no more than $\left\lfloor\alpha_{i}^{\prime}\right\rfloor$ other members. As with the partition $P(\alpha), i$ has no congestion cost with the partition $P\left(\alpha^{\prime}\right)$. However, because $i$ is in a smaller club when he changes his reported type from $\alpha_{i}$ to $\alpha_{i}^{\prime}$, his share of the facility cost increases. Hence, reporting $\alpha_{i}^{\prime}$ is not beneficial for him. If, however, $\alpha_{i}>\alpha_{i}^{\prime} \geq n_{i}\left(S_{i}(P(\alpha))\right)$, then the original partition $P(\alpha)$ is cost efficient when the reported type vector is $\alpha^{\prime}$. Moreover, $\mathscr{E}\left(\alpha^{\prime}\right) \subseteq \mathscr{E}(\alpha)$. As a consequence, the serial dictatorship chooses the same partition when the reported type profile is $\alpha^{\prime}$ as it does when it is $\alpha$. 
(ii) Suppose that $\alpha_{i}^{\prime}>\alpha_{i}$. Increasing $\alpha_{i}$ raises the threshold at which i's congestion cost becomes positive. Consequently, reporting $\alpha_{i}^{\prime}$ instead of $\alpha$ does not change the aggregate cost associated with any of the partitions in $\mathscr{E}(\alpha)$. There are four cases to consider.

First, suppose that $n_{i}\left(S_{i}(P(\alpha))\right)>n_{i}\left(S_{i}\left(P\left(\alpha^{\prime}\right)\right)\right)$. In this case, by reporting $\alpha_{i}^{\prime}$ instead of $\alpha_{i}$, there is no change in $i$ 's congestion cost (it remains 0 ), but his share of the facility's financial cost increases, so reporting $\alpha_{i}^{\prime}$ makes $i$ worse off.

Second, suppose that $n_{i}\left(S_{i}(P(\alpha))\right)=n_{i}\left(S_{i}\left(P\left(\alpha^{\prime}\right)\right)\right)$. In this case, $i$ is indifferent between $P(\alpha)$ and $P\left(\alpha^{\prime}\right)$, and so has no incentive to misreport.

Third, suppose that $n_{i}\left(S_{i}\left(P\left(\alpha^{\prime}\right)\right)\right)>\left\lfloor\alpha_{i}+1\right\rfloor$. In this case, by reporting $\alpha_{i}^{\prime}$ instead of $\alpha_{i}$, i's congestion cost increases to $c$ from 0 . Because $c>1$, the increase in his congestion cost exceeds the reduction in his share of the financial cost of the facility, and therefore he is worse off if he reports $\alpha_{i}^{\prime}$.

Finally, suppose that $n_{i}\left(S_{i}(P(\alpha))\right)<n_{i}\left(S_{i}\left(P\left(\alpha^{\prime}\right)\right)\right) \leq\left\lfloor\alpha_{i}+1\right\rfloor$. In this case, because $n_{i}\left(S_{i}\left(P\left(\alpha^{\prime}\right)\right)\right) \leq\left\lfloor\alpha_{i}+1\right\rfloor$, $i$ 's congestion cost remains at 0 if he reports $\alpha_{i}^{\prime}$ instead of $\alpha_{i}$. Because the congestion costs of the other individuals do not depend on $i$ 's type and $P\left(\alpha_{i}^{\prime}\right)$ minimizes aggregate costs when the type vector is $\alpha_{i}^{\prime}$, it then follows that the aggregate cost when the partition is $P\left(\alpha_{i}^{\prime}\right)$ is the same as it is with $P\left(\alpha_{i}\right)$. Thus, $P\left(\alpha_{i}^{\prime}\right) \in \mathscr{E}(\alpha) \subseteq \mathscr{E}\left(\alpha^{\prime}\right)$. Because $P\left(\alpha_{i}^{\prime}\right) \in \mathscr{E}(\alpha)$ but is not chosen by the serial dictatorship when $\alpha$ is reported, it is not chosen when $\alpha_{i}^{\prime}$ is reported either. Hence, it is not possible for this case to occur.

\section{Strategy-Proofness and the Other Axioms}

In the preceding section, we have shown that a strategy-proof allocation rule cannot be cost efficient if the congestion cost function is strictly increasing in the type parameter. In this section, we provide an example that demonstrates the possibility of constructing an allocation rule that satisfies all of our axioms except for Cost Efficiency when $n=3$. To simplify the discussion, we suppose that congestion costs are linear and that the cost of a club facility is shared equally among the club members. ${ }^{6}$

In Example 1, we show that it is possible for an allocation rule to satisfy both Nondictatorship and Individual Rationality in addition to Strategy-Proofness and Pareto Optimality.

Example 1. Let $N=\{1,2,3\}$ and suppose that there are linear congestion costs and equal cost-sharing.

To define the allocation rule, we first partition the type space. The cube $\left[0, \frac{1}{2}\right]^{3}$ is split into five subsets. The set $\left[0, \frac{1}{2}\right)^{3}$ obtained by removing the upper faces of this cube is subdivided into two sets, $T_{1}$ and $T_{2}$. One of upper edges of this cube is the set $T_{4}$ and two of the other upper edges constitute the set $T_{5}$. The set $T_{3}$ consists of

\footnotetext{
${ }^{6}$ In the examples in this and the following section, it does not matter if the congestion cost function is strictly increasing in the type parameter or if, instead, it is merely non-decreasing.
} 
the rest of the three upper faces. The rest of the parameter space is subdivided into the sets $T_{6}$ and $T_{7}$. Letting $i, j$, and $k$ with $i \neq j \neq k \neq i$ be any relabelling of the three individuals, this partition is formally defined as follows.

$$
\begin{aligned}
& T_{1}=\left\{\alpha \in \mathbb{R}_{+}^{3} \mid 0 \leq \alpha_{1} \leq \frac{1}{6}, 0 \leq \alpha_{2} \leq \frac{1}{3}, \text { and } 0 \leq \alpha_{3} \leq \frac{1}{6}\right\} ; \\
& T_{2}=\left\{\alpha \in \mathbb{R}_{+}^{3} \mid 0 \leq \alpha_{1}<\frac{1}{2}, 0 \leq \alpha_{2}<\frac{1}{2}, \text { and } 0 \leq \alpha_{3}<\frac{1}{2},\right\} \backslash T_{1} ; \\
& T_{3}=\left\{\alpha \in \mathbb{R}_{+}^{3} \mid 0 \leq \alpha_{i}<\frac{1}{2}, 0 \leq \alpha_{j}<\frac{1}{2}, \text { and } \alpha_{k}=\frac{1}{2}\right\} ; \\
& T_{4}=\left\{\alpha \in \mathbb{R}_{+}^{3} \mid \alpha_{1}=\alpha_{3}=\frac{1}{2} \text { and } 0 \leq \alpha_{2} \leq \frac{1}{2}\right\} ; \\
& T_{5}=\left\{\alpha \in \mathbb{R}_{+}^{3} \mid\left[\alpha_{1}=\alpha_{2}=\frac{1}{2} \text { and } 0 \leq \alpha_{3} \leq \frac{1}{2}\right] \text { or }\left[\alpha_{2}=\alpha_{3}=\frac{1}{2} \text { and } 0 \leq \alpha_{1} \leq \frac{1}{2}\right]\right\} ; \\
& T_{6}=\left\{\alpha \in \mathbb{R}_{+}^{3} \mid 0 \leq \alpha_{i} \leq \frac{1}{2}, 0 \leq \alpha_{j} \leq \frac{1}{2}, \text { and } \frac{1}{2}<\alpha_{k}\right\} ; \\
& T_{7}=\left\{\alpha \in \mathbb{R}_{+}^{3} \mid \frac{1}{2}<\alpha_{i} \text { and } \frac{1}{2}<\alpha_{j}\right\} ;
\end{aligned}
$$

The allocation rule $A$ is defined by setting:

$$
\begin{aligned}
& P(\alpha)=\{\{1,2,3\}\} \text { if } \alpha \in T_{1} ; \\
& P(\alpha)=\{\{1,3\},\{2\}\} \text { if } \alpha \in T_{2} ; \\
& P(\alpha)=\{\{k\},\{i, j\}\} \text { if } \alpha \in T_{3} ; \\
& P(\alpha)=\{\{1,2\},\{3\}\} \text { if } \alpha \in T_{4} ; \\
& P(\alpha)=\{\{1,3\},\{2\}\} \text { if } \alpha \in T_{5} ; \\
& P(\alpha)=\{\{k\},\{i, j\}\} \text { if } \alpha \in T_{6} ; \\
& P(\alpha)=\{\{1\},\{2\},\{3\}\} \text { if } \alpha \in T_{7} ;
\end{aligned}
$$

We now show that $A$ satisfies Strategy-Proofness, Pareto Optimality, Nondictatorship, and Individual Rationality.

Strategy-Proofness. First, suppose that $\alpha \in T_{1}$. In this case, $\{1,2,3\}$ is an optimal club for both 1 and 3 , so they have no incentive to misreport their types. The same is true for 2 if $0 \leq \alpha_{2} \leq \frac{1}{6}$. If $\frac{1}{6}<\alpha_{2} \leq \frac{1}{3}, 2$ would only be better off in a two-member club. This outcome only occurs if the type report is in $T_{3}$ with $k \neq 2, T_{4}$, or $T_{6}$ with $k \neq 2$. Each of these three situations requires someone other than 2 to announce a type weakly greater than $\frac{1}{2}$. But this is not possible with a non-truthful report on the part of 2 alone.

Second, suppose that $\alpha \in T_{2}$. There are two cases to consider.

(i) Suppose that $0 \leq \alpha_{2} \leq \frac{1}{3}$. Then, at least one of $\alpha_{1}$ or $\alpha_{3}$ must be greater than $\frac{1}{6}$, otherwise $\alpha$ would be in $T_{1}$. Without loss of generality, suppose that $\frac{1}{6}<\alpha_{1}<\frac{1}{2}$. For $1,\{1,3\}$ is an optimal club. If it is the case that $\frac{1}{6}<\alpha_{3} \leq \frac{1}{2}$, then $\{1,3\}$ is also 
an optimal club for 3 . If, however, $0 \leq \alpha_{3}<\frac{1}{6}, 3$ can only be made better off by switching to the three-person club, which requires the reported type vector to be in $T_{1}$. Because $\frac{1}{6}<\alpha_{1}<\frac{1}{2}$, this is not possible using a unilateral deviation from a truthful report by 3 . As far as 2 is concerned, he either strictly or weakly prefers being in the three-member club or in a two-member club to being in his own singlemember club. It is not possible for 2 to report a type that results in the type vector being in $T_{1}$ because $\frac{1}{6}<\alpha_{1}<\frac{1}{2}$, and so the three-member club is not achievable. Individual 2 is assigned to a two-member club only if the reported type is in $T_{3}$ with $k \neq 2, T_{4}$, or $T_{6}$ with $k \neq 2$. As in the case of $T_{1}$, it is not possible for the reported type vector to be in any of these three sets with a unilateral deviation from a truthful report by 2 .

(ii) Now, suppose that $\frac{1}{3}<\alpha_{2}<\frac{1}{2}$. The optimal club size for both 1 and 3 is either two or three. They are in the same two-person club. Because $\alpha_{2}>\frac{1}{3}$, a unilateral change in the reports of either 1 or 3 cannot result in a reported type vector in $T_{1}$, which is what is required to obtain the three-member club. Hence, neither of them has a beneficial manipulation. Individual 2 prefers being in his own single-member club to the three-person club, so can only be made better off by misreporting so that the reported type vector is in $T_{3}$ with $k \neq 2, T_{4}$, or $T_{6}$ with $k \neq 2$, which is not possible with a unilateral deviation from a truthful report by 2 .

Third, suppose that $\alpha$ is in either $T_{3}$ or $T_{6}$. In both of these cases, $k$ is in an optimal club. If $\frac{1}{6} \leq \alpha_{i} \leq \frac{1}{2}$, then $i$ is also in an optimal club. If $0 \leq \alpha_{i}<\frac{1}{6}, i$ would only prefer being in the three-member club, which requires the reported type vector to be in $T_{1}$. Because $\alpha_{k} \geq \frac{1}{2}$, it is not possible for the reported type vector to be in $T_{1}$ by a unilateral deviation from a truthful report by $i$. The same reasoning shows that $j$ cannot beneficially manipulate the outcome either.

Fourth, suppose that $\alpha$ is in either $T_{4}$ or $T_{5}$. In this case, everybody is in an optimal club except for any individual $i$ for which $0 \leq \alpha_{i}<\frac{1}{6}$. If that is the case, $i$ is in a two-member club and only prefers being in the three-member club. That outcome requires the reported type vector to be in $T_{1}$, which is not possible with a unilateral deviation from a truthful report by $i$ because the types of the other two individuals are both equal to $\frac{1}{2}$.

Finally, suppose that $\alpha \in T_{7}$. In this case, both $i$ and $j$ are assigned their optimal clubs. The allocation is independent of $k$ 's report, so $k$ has no opportunity to manipulate the allocation.

Pareto Optimality. First, suppose that $\alpha \in T_{1}$. In this case, $\{1,2,3\}$ is the unique optimal club for anybody whose type is less than $\frac{1}{6}$, in which case the allocation is Pareto optimal. If $\alpha_{1}=\alpha_{3}=\frac{1}{6}$, then both individuals 1 and 3 are indifferent between being in the club $\{1,2,3\}$ and being in a two-member club, which is only possible if the partition is $\{\{1,3\},\{2\}\}$. But with the club $\{3\}, 2$ is worse off if $\alpha_{2} \neq \frac{1}{3}$ and indifferent to the change if $\alpha_{2}=\frac{1}{3}$. So, no Pareto improvement is possible.

Second, suppose that $\alpha \in T_{2}$. We consider two cases.

(i) Suppose that $0 \leq \alpha_{2} \leq \frac{1}{3}$. Then, at least one of $\alpha_{1}$ or $\alpha_{3}$ must be greater than $\frac{1}{6}$, otherwise $\alpha$ would be in $T_{1}$. Without loss of generality, suppose that that this is the case for 1 . Then, $\{1,3\}$ is an optimal club for 1 . He is indifferent between being 
in $\{1,3\}$ or $\{1,2\}$. However, in the latter case, 3 is in the single-member club $\{3\}$, which makes him worse off than with the club $\{1,3\}$ because $0 \leq \alpha_{3}<\frac{1}{2}$.

(ii) Now, suppose that $\frac{1}{3}<\alpha_{2}<\frac{1}{2}$. In this case, 2 strictly prefers his singlemember club $\{2\}$ to $\{\{1,2,3\}\}$. The other partitions are $\{\{1,2\},\{3\}\},\{\{2,3\},\{1\}\}$, and $\{\{1\},\{2\},\{3\}\}$, all of which make either 1 or 3 worse off.

Third, suppose that $\alpha \in T_{3}$. Then, the partition is $\{\{k\},\{i, j\}\}$. Individual $k$ is in an optimal club and is indifferent between being a single-member or two-member club. Thus, in order not to make him worse off, the partition can only be changed to $\{\{i\},\{j\},\{k\}\},\{\{k, i\},\{j\}\}$, or $\{\{k, j\},\{i\}\}$. In all of these partitions, either $i$ or $j$ is worse off than in $\{\{k\},\{i, j\}\}$.

Fourth, suppose that $\alpha$ is in either $T_{4}$ or $T_{5}$. In this case, everybody is in an optimal club except for any individual $i$ for which $0 \leq \alpha_{i}<\frac{1}{6}$. If that is the case, $i$ is only better off with the three-member club, but that would make the other two individuals worse off.

Fifth, suppose that $\alpha \in T_{6}$. In this case, $\{k\}$ is $k$ 's unique optimal club, so the allocation is Pareto optimal.

Finally, suppose that $\alpha \in T_{7}$. In this case, both $i$ and $j$ are assigned their unique optimal clubs, so the allocation is Pareto optimal.

Nondictatorship. Consider any individual $i$. If $i$ were a dictator and $0 \leq \alpha_{i}<$ $\frac{1}{6}$, then the partition would be $\{\{1,2,3\}\}$. However, if the other two individuals have types that exceed $\frac{1}{2}$, the type vector is in $T_{7}$ and the partition that is chosen is $\{\{1\},\{2\},\{3\}\}$. Hence, nobody is a dictator.

Individual Rationality. We only need to consider clubs with two or three members. If $\alpha \in T_{1}$, then $\alpha_{i} \leq \frac{1}{3}$ for all $i$, and so nobody is better off in his own singlemember club than with the partition $\{\{1,2,3\}\}$. If $\alpha \in T_{2}$, then $0 \leq \alpha_{1}<\frac{1}{2}$ and $0 \leq \alpha_{1}<\frac{1}{2}$ and, therefore, neither 1 nor 2 prefer being in his own single-member club to the partition $\{\{1,3\},\{2\}\}$. The same argument holds for $i$ and $j$ in $T_{3}$ and $T_{6}$, for 1 and 2 in $T_{4}$, and for 1 and 3 in $T_{5}$. Hence, $A$ satisfies Individual Rationality.

\section{Further Examples}

We have not been able to find a way to extend the rule constructed in Example 1 for $n>3$. This raises the issue of whether there are other more transparent rules that satisfy Strategy-Proofness, Pareto Optimality, Individual Rationality, and Nondictatorship. An obvious candidate is a serial dictatorship. A serial dictatorship is strategy-proof and always chooses a weakly Pareto optimal allocation (i.e., there is no other allocation that makes everyone better off). Unfortunately, it need not choose allocations that are strictly Pareto optimal. To illustrate this claim, Example 2 describes an allocation rule that employs a form of serial dictatorship but does not satisfy Pareto Optimality. It also does not satisfy either Nondictatorship or Individual Rationality. 
Example 2. Assume that congestion costs are linear and that costs are shared equally. For each type vector, the allocation is constructed as follows. First, individual 1's optimal club size is determined. Using the natural order of individuals, individuals are assigned to a club that includes individual 1 until this optimal size is achieved. Once this is done, if there are unassigned individuals, the process is sequentially repeated using the optimal club size of the next person in line until everybody is in a club. If there are two optimal club sizes at any step in this procedure, the tie is broken in favor of the smaller one.

Individual $i$ 's preferences are only taken into account if they are used to determine the size of $i$ 's club. Because this size is optimal for $i$, this allocation rule satisfies Strategy-Proofness.

To show that our other axioms are violated, consider the case in which $n=3 .{ }^{7} \mathrm{Be}-$ ing a serial dictatorship, the chosen allocations are weakly Pareto optimal. However, as we now show, this allocation rule does not satisfy Pareto Optimality. Suppose that 1 's unique optimal group size is two, so that the clubs $\{1,2\}$ and $\{3\}$ are formed. Further suppose that 2's unique optimal club size is one, whereas for 3 it is two. Then, the partition $\{\{1,3\},\{2\}\}$ Pareto dominates $\{\{1,2\},\{3\}\}$. If $0 \leq \alpha_{1}<\frac{1}{6}$, only a single club is formed. If, however, $\alpha_{2}$ and $\alpha_{3}$ both exceed $\frac{1}{2}$, they would prefer to be in their own single-member clubs. Hence, this rule violates Individual Rationality. It clearly violates Nondictatorship.

The serial dictatorship in Example 2 fails to satisfy Pareto Optimality because only one person's preferences are considered when forming each club. As Example 3 demonstrates, it is possible for a serial dictatorship to satisfy both StrategyProofness and Pareto Optimality by taking account of the other individuals' preferences. However, the allocation rule we construct violates both Nondictatorship and Individual Rationality.

Example 3. Let $N=\{1,2,3\}$. Suppose that there are linear congestion costs and equal cost-sharing.

The allocation rule is a serial dictatorship using the individual preferences over allocations, with individual 1 being the first dictator and indifferences broken by individuals 2 and 3 in that order. If a unique partition has not been chosen after all three individuals have been considered, a linear order $\succ$ of the partitions in $\mathscr{P}$ is used to make a final choice from the partitions that are still under consideration. For concreteness, we assume that $\succ$ is given by $\{\{1\},\{2\},\{3\}\} \succ\{\{1\},\{2,3\}\} \succ$ $\{\{1,3\},\{2\}\} \succ\{\{1,2\},\{3\}\} \succ\{\{1,2,3\}\}$.

To state this rule formally, we partition the type space into the following 18 sets. The values of the type parameter used to define the partition are $\frac{1}{6}, \frac{1}{3}$, and $\frac{1}{2}$. These are the values of the type parameter for which an individual is indifferent between being in a club with (i) two or three members, (ii) one or three members, and (iii) one or two members, respectively.

$$
T_{1}=\left\{\alpha \in \mathbb{R}_{+}^{3} \mid \alpha_{1}>\frac{1}{2} \text { and } \alpha_{2}>\frac{1}{2}\right\}
$$

\footnotetext{
${ }^{7}$ Our arguments can be adapted to apply to larger values of $n$.
} 


$$
\begin{aligned}
& T_{2}=\left\{\alpha \in \mathbb{R}_{+}^{3} \mid \alpha_{1}>\frac{1}{2}, \alpha_{2}=\frac{1}{2}, \text { and } \alpha_{3} \geq \frac{1}{2}\right\} ; \\
& T_{3}=\left\{\alpha \in \mathbb{R}_{+}^{3} \mid \alpha_{1}>\frac{1}{2}, \alpha_{2}=\frac{1}{2}, \text { and } 0 \leq \alpha_{3}<\frac{1}{2}\right\} ; \\
& T_{4}=\left\{\alpha \in \mathbb{R}_{+}^{3} \mid \alpha_{1}=\frac{1}{2}, \alpha_{2} \geq \frac{1}{2}, \text { and } \alpha_{3} \geq \frac{1}{2}\right\} ; \\
& T_{5}=\left\{\alpha \in \mathbb{R}_{+}^{3} \mid \alpha_{1}=\frac{1}{2}, \alpha_{2}>\frac{1}{2}, \text { and } 0 \leq \alpha_{3}<\frac{1}{2}\right\} ; \\
& T_{6}=\left\{\alpha \in \mathbb{R}_{+}^{3} \mid \alpha_{1}=\frac{1}{2}, \alpha_{2}=\frac{1}{2}, \text { and } 0 \leq \alpha_{3}<\frac{1}{2}\right\} ; \\
& T_{7}=\left\{\alpha \in \mathbb{R}_{+}^{3} \mid \frac{1}{6}<\alpha_{1}<\frac{1}{2} \text { and } \alpha_{2}>\frac{1}{2}\right\} ; \\
& T_{8}=\left\{\alpha \in \mathbb{R}_{+}^{3} \mid \frac{1}{6}<\alpha_{1}<\frac{1}{2}, \alpha_{2}=\frac{1}{2}, \text { and } \alpha_{3}>\frac{1}{2}\right\} ; \\
& T_{9}=\left\{\alpha \in \mathbb{R}_{+}^{3} \mid \frac{1}{6}<\alpha_{1}<\frac{1}{2}, \alpha_{2}=\frac{1}{2}, \text { and } 0 \leq \alpha_{3}=\frac{1}{2}\right\} ; \\
& T_{10}=\left\{\alpha \in \mathbb{R}_{+}^{3} \mid \frac{1}{6}<\alpha_{1}<\frac{1}{2} \text { and } 0 \leq \alpha_{2}<\frac{1}{2}\right\} ; \\
& T_{11}=\left\{\alpha \in \mathbb{R}_{+}^{3} \mid \alpha_{1}=\frac{1}{6} \text { and } \alpha_{2}>\frac{1}{2}\right\} ; \\
& T_{12}=\left\{\alpha \in \mathbb{R}_{+}^{3} \mid \alpha_{1}=\frac{1}{6}, \alpha_{2}=\frac{1}{2}, \text { and } \alpha_{3}>\frac{1}{2}\right\} ; \\
& T_{13}=\left\{\alpha \in \mathbb{R}_{+}^{3} \mid \alpha_{1}=\frac{1}{6}, \alpha_{2}=\frac{1}{2}, \text { and } \alpha_{3} \leq \frac{1}{2}\right\} ; \\
& T_{14}=\left\{\alpha \in \mathbb{R}_{+}^{3} \mid \alpha_{1}=\frac{1}{6} \text { and } \frac{1}{6}<\alpha_{2}<\frac{1}{2}\right\} ; \\
& T_{15}=\left\{\alpha \in \mathbb{R}_{+}^{3} \mid \alpha_{1}=\frac{1}{6}, \alpha_{2}=\frac{1}{6} \text {, and } \alpha_{3} \geq \frac{1}{3}\right\} ; \\
& T_{16}=\left\{\alpha \in \mathbb{R}_{+}^{3} \mid \alpha_{1}=\frac{1}{6}, \alpha_{2}=\frac{1}{6}, \text { and } \alpha_{3}<\frac{1}{3}\right\} ; \\
& T_{17}=\left\{\alpha \in \mathbb{R}_{+}^{3} \mid \alpha_{1}=\frac{1}{6} \text { and } 0 \leq \alpha_{2}<\frac{1}{6}\right\} ; \\
& T_{18}=\left\{\alpha \in \mathbb{R}_{+}^{3} \mid 0 \leq \alpha_{1}<\frac{1}{6}\right\} \text {. }
\end{aligned}
$$

The allocation rule $A$ is formally defined as follows:

$$
\begin{aligned}
& P(\alpha)=\{\{1\},\{2\},\{3\}\} \text { if } \alpha \in T_{1} \cup T_{2} \cup T_{4} ; \\
& P(\alpha)=\{\{1\},\{2,3\}\} \text { if } \alpha \in T_{3} \cup T_{6} ; \\
& P(\alpha)=\{\{1,2\},\{3\}\} \text { if } \alpha \in T_{8} \cup T_{10} \cup T_{12} \cup T_{14} \cup T_{15} ;
\end{aligned}
$$




$$
\begin{aligned}
& P(\alpha)=\{\{1,3\},\{2\}\} \text { if } \alpha \in T_{5} \cup T_{7} \cup T_{9} \cup T_{11} \cup T_{13} \\
& P(\alpha)=\{\{1,2,3\}\} \text { if } \alpha \in T_{16} \cup T_{17} \cup T_{18} .
\end{aligned}
$$

A complete proof of the claim that the informal definition of the allocation rule is being used to construct $A$ is rather lengthy, and so we only verify it for a few of the sets in the partition of the type space. The sets considered have been chosen to illustrate differences in the way that this serial dictatorship operates in different regions of the type space.

First, suppose that $\alpha \in T_{1}$. Because $\alpha_{1}>\frac{1}{2}$ and 1 is the first dictator, 1 must be in a single-member club. This individual is indifferent about how 2 and 3 partition themselves into clubs. Because $\alpha_{2}>\frac{1}{2}$ and 2 is the second dictator, 2 must be in a single-member club, so that leaves no choice but for 3 to be in a single-member club as well.

Second, suppose that $\alpha \in T_{2}$. As in the previous case, 1 must be in a singlemember club. Now, however, 2 is indifferent between being in a club with 3 or not, so we consider 3's preferences. If $\alpha_{3}>\frac{1}{2}, 3$ prefers that 2 and 3 be in separate clubs, so this is chosen. If $\alpha_{3}=\frac{1}{2}, 3$ is indifferent between the two options. The final tie-breaking rule then assigns 2 and 3 to separate clubs.

Third, suppose that $\alpha \in T_{3}$. As in the previous case, 1 must be in a single-member club and 2 is indifferent between being in a club with 3 or not. Because $\alpha_{3}<\frac{1}{2}$, 3 strictly prefers being in a two-member club to being in a single-member club. Consequently, 2 and 3 form a club.

Fourth, suppose that $\alpha \in T_{4}$. Now, 1 is indifferent between a single-member club and a club with one other member. If $\alpha_{2}>\frac{1}{2}, 2$ chooses to be in a single-member club. If $\alpha_{3}>\frac{1}{2}$, so does 3 , with the consequence that there are three clubs. If, instead, $\alpha_{3}=\frac{1}{2}$, the last tie-breaking rule also results in three clubs being chosen. If $\alpha_{2}=\frac{1}{2}$ and $\alpha_{3}>\frac{1}{2}$, then both 1 and 2 are indifferent between a single-member club and a club with one other member, whereas 3 prefers a single-member club. Individual 3 's choice is implemented, and then the final tie-breaking rule assigns 1 and 2 to separate clubs. If, however, $\alpha_{1}=\alpha_{2}=\alpha_{3}=\frac{1}{2}$, all three individuals are indifferent between being in a single-member or a two-member club. Applying the final tiebreaking rule also results in three separate clubs being chosen.

Finally, suppose that $\alpha \in T_{15} \cup T_{16}$. In this case, individuals 1 and 2 are indifferent between being in a two-member or three-member club. Thus, the partitions 3 gets to choose from are $\{\{1,2,3\}\}$ and $\{\{1,2\},\{3\}\}$. As previously noted, 3 would choose the former if $\alpha_{3}<\frac{1}{3}$ and the latter if $\alpha_{3}>\frac{1}{3}$. If $\alpha_{3}=\frac{1}{3}$, then 3 is indifferent between these two partitions, in which case the final tie-breaking rule chooses $\{\{1,2\},\{3\}\}$.

With the allocation rule $A$, when an individual chooses, he leaves for further consideration all of the partitions that he most prefers from among the partitions that are still available when it is his turn to choose. If there is more than one partition remaining after he chooses, this is only because he is indifferent between them. As a consequence, $A$ satisfies Strategy-Proofness and Pareto Optimality. Individual 1 is a dictator, so $A$ does not satisfy Nondictatorship. If $\alpha \in T_{7}$ and $\alpha_{3}>\frac{1}{2}$, individual 3 
is assigned to the club $\{1,3\}$, but strictly prefers being on his own. Hence, $A$ does not satisfy Individual Rationality.

Examples 1,2, and 3 show that the construction of strategy-proof rules satisfying Pareto Optimality, Nondictatorship, and Individual Rationality is a nontrivial task. This may seem to be inconsistent with the fact that Long (2019) has provided two rules for choosing the membership of a single club that are (weakly group) strategyproof, Pareto optimal, and do not force anybody to join the club. ${ }^{8}$ However, in fact, there is no inconsistency because, in contrast to Long, we require everybody be in some club. To show why this matters, we consider her voting on ascending size rule for choosing a single club's size. We define an iterative version of this procedure that partitions the individuals into clubs and show that it is not strategy-proof when costs are shared equally.

Here is an informal description of Long's voting rule. Starting with group size 1, for each club size $k \geq 1$ in turn, individuals vote on whether they prefer to be in a club of that size or to remain on their own. For Long, this is a club of size 0 . If more than $k$ individuals vote in favor of a club with $k$ members, the procedure continues with a vote for a club of size $k+1$ unless sufficiently many individuals according to a priority ordering used to breaks ties over club membership prefer a smaller size. However, in Long's framework, only one club forms and individuals are allowed not to join any club at all. In contrast, everyone must be in a club in our framework. This turns out to be an important difference.

Our club partitioning rule employs an iterative version of Long's voting procedure in which, for each type vector, one club at a time selected. Because we do not permit a club of size 0 , voting in our case starts with club size $k=2$ and individuals are asked to vote on whether they prefer to be in a club of size $k$ to one of size 1 .

Before formally stating our rule, some further notation is needed. Let $\sigma$ be a fixed linear priority ordering on $N$, where $i \sigma j$ denotes that $i$ has higher priority than $j$. For all $\alpha \in \mathbb{R}_{+}^{n}$ and all $k \in\{2, \ldots, n\}$, let $\Delta_{N_{l}, k}(\alpha)$ be the set of individuals in $N_{l} \subseteq N$ who strictly prefer club size $k$ to club size 1 . If $\left|\Delta_{N_{l}, k}(\alpha)\right| \geq k$, let $\tilde{\Delta}_{N_{l}, k}^{\sigma}(\alpha)$ be the first $k$ members of $\Delta_{N_{l}, k}(\alpha)$ according to $\sigma$. For $k=1$, let $\tilde{\Delta}_{N_{l}, k}^{\sigma}(\alpha)$ be the first person in $N_{l}$ according to $\sigma$.

Iterative Voting on Ascending Size for the Priority Ordering $\sigma$. A club partitioning rule $P^{\sigma}$ is Iterative Voting on Ascending Size for the Priority Ordering $\sigma$ if for all $\alpha \in \mathbb{R}_{+}^{n}, P^{\sigma}(\alpha)$ is obtained using the following two iterative algorithms.

Step $l$ of the refinement algorithm determines the $l$ th club $C_{l}(\alpha)$ in the partition $P^{\sigma}(\alpha)$ by applying the club size algorithm described below to the set of individuals $N_{l}=N \backslash\left(\cup_{q=1}^{l-1} C_{q}(\alpha)\right)$. The algorithm begins with $l=1$ and terminates when $C_{l}(\alpha)=N_{l}$. For $l \geq 2$, at the end of Step $l-1, N$ has been partitioned into $\left\{C_{1}(\alpha), \ldots, C_{l-1}(\alpha), N_{l}\right\}$. In Step $l$, this partition is refined by partitioning $N_{l}$ into one or two sets.

\footnotetext{
${ }^{8}$ There is no explicit cost sharing rule. Cost sharing is implicit in the preferences over club size.
} 
The club size algorithm applied to the nonempty set of individuals $N_{l} \subseteq N$ identifies the club $C_{l}(\alpha) \subseteq N_{l}{ }^{9}$ In Step 1 , if $\left|N_{l}\right|=1$, set $C_{l}(\alpha)=\tilde{\Delta}_{N_{l}, k}^{\sigma}(\alpha)$ (which equals $N_{l}$ ) and terminate the algorithm; otherwise proceed to Step $k=2$. Step $k$ for $k \geq 2$ is defined by the following four cases.

Case 1 If $\left|\Delta_{N_{l}, k}(\alpha)\right|<k$, stop and set $C_{l}(\alpha)=\tilde{\Delta}_{N_{l}, k}^{\sigma}(\alpha)$.

Case 2 If $\left|\Delta_{N_{l}, k}(\alpha)\right| \geq k$ and at least $\left|\Delta_{N_{l}, k}(\alpha)\right|-k+1$ individuals in $\Delta_{N_{l}, k}(\alpha) \cap$ $\tilde{\Delta}_{N_{l}, k-1}^{\sigma}(\alpha)$ strictly prefer club size $k-1$ to size $k$, then stop and set $C_{l}(\alpha)=$ $\tilde{\Delta}_{N_{l}, k-1}^{\sigma, k}(\alpha)$.

Case 3 If $\left|\Delta_{N_{l}, k}(\alpha)\right|=k$ and nobody in $\Delta_{N_{l}, k}(\alpha) \cap \tilde{\Delta}_{N_{l}, k-1}^{\sigma}(\alpha)$ strictly prefers club size $k-1$ to size $k$, then stop and set $C_{l}(\alpha)=\Delta_{N_{l}, k}^{\sigma}(\alpha)$.

Case 4 If $\left|\Delta_{N_{l}, k}(\alpha)\right|>k$ and fewer than $\left|\Delta_{N_{l}, k}(\alpha)\right|-k+1$ individuals in $\Delta_{N_{l}, k}(\alpha) \cap$ $\tilde{\Delta}_{N_{l}, k-1}^{\sigma}(\alpha)$ strictly prefer club size $k-1$ to size $k$, then go to Step $k+1$.

In Example 4, we show that if there is equal cost sharing, then choosing club partitions using the iterative voting rule described above is not strategy-proof. Hence, a natural extension of Long's iterative voting rule for determining the membership of a single club to the club partitioning problem does not preserve truth-telling as a dominant strategy.

Example 4. Let $N=\{1,2,3,4\}$ and suppose that there is equal cost-sharing. Iterative voting on ascending size for the priority ordering $\sigma$ given by $1 \sigma 2 \sigma 3 \sigma 4$ is used to determine the partition $P^{\sigma}(\alpha)$ for all $\alpha \in \mathbb{R}_{+}^{4}$. For individual $i$ of type $\alpha_{i}$, let $k \succ_{\alpha_{i}} k^{\prime}$ denote that $i$ strictly prefers club size $k$ to $k^{\prime}$.

Consider a type vector $\alpha \in \mathbb{R}_{+}^{4}$ for which $2 \succ_{\alpha_{1}} 3 \succ_{\alpha_{1}} 1 \succ_{\alpha_{1}} 4$ and $3 \succ_{\alpha_{j}} 2 \succ_{\alpha_{j}}$ $1 \succ \alpha_{j} 4$ for $j \neq 1$. The club size algorithm for identifying $C_{1}(\alpha)$ terminates in Step 4. Case 1 applies, so the three individuals with the highest priority form the first club. Hence, $C_{1}(\alpha)=\{1,2,3\}$. There is only one person remaining, so $P^{\sigma}(\alpha)$ is completed with the club $C_{2}(\alpha)=\{4\}$.

Suppose that individual 4 instead reports a type $\alpha_{4}^{\prime}$ for which $2 \succ_{\alpha_{4}^{\prime}} 1 \succ_{\alpha_{4}^{\prime}} 3 \succ_{\alpha_{4}^{\prime}}$ 4. Let $\alpha^{\prime}=\left(\alpha_{1}, \alpha_{2}, \alpha_{3}, \alpha_{4}^{\prime}\right)$. With this type report, the club size algorithm for identifying $C_{1}\left(\alpha^{\prime}\right)$ terminates in Step 3. Case 2 applies, so the two individuals with the highest priority form the first club. Hence, $C_{1}\left(\alpha^{\prime}\right)=\{1,2\}$. The club size algorithm is then repeated for $N_{2}=\{3,4\}$. It terminates in Step 2 with Case 3 applying, so $C_{2}\left(\alpha^{\prime}\right)=\{3,4\}$, which completes the partition.

Because $2 \succ_{\alpha_{j}} 1$, at $\alpha$, individual 4 has an incentive to misreport his type as $\alpha_{4}^{\prime}$. Hence, this allocation rule is not strategy-proof.

\section{Concluding Remarks}

We have shown that no allocation rule is strategy-proof and cost efficient when congestion costs are strictly increasing in the type parameter, but that these properties

${ }^{9}$ For a discussion of this algorithm, see Long (2019). 
are compatible if congestion costs are dichotomous. We have also shown that when $n=3$, it is possible to construct a strategy-proof allocation rules with equal cost sharing that is Pareto optimal, nondictatorial, and individually rational when the congestion cost is linear in the type parameter.

The are a number of possible extensions of our analysis that are worth exploring. For example, it would be interesting to provide characterizations of all of the allocation rules that satisfy Strategy-Proofness, Pareto optimality, Nondictatorship, and Individually Rationality, both with and without the assumptions of equal cost sharing and linear congestion costs. It would also be of interest to allow for the size of a club good facility to be determined endogenously.

\section{Acknowledgements}

We dedicate this article to Semih Koray. Semih was one of the first Turkish scholars to be recognized internationally for his contributions to the design of economic mechanisms. His scholarship and his mentoring of students and junior faculty has played a fundamental role in the establishment of a community of Turkish scholars, now spread world wide, whose research focuses on mechanism and market design. Semih's kindness and generosity are legendary.

\section{References}

Bloch F, Dutta B (2011) Formation of networks and coalitions. In: Benhabib J, Jackson MO, Bisin A (eds) Handbook of Social Economics, vol 1A, North-Holland, San Diego, pp 729-779

Bogomolnaia A, Jackson MO (2002) The stability of hedonic coalition structures. Games Econ Behav 38:201-230

Bogomolnaia A, Nicolò A (2005) Stable assignment of public facilities under congestion. J Public Econ Theory 7:65-91

Buchanan JM (1965) An economic theory of clubs. Economica 32:1-14

Cornes R, Sandler T (1996) The Theory of Externalities, Public Goods, and Club Goods, 2nd edn. Cambridge University Press, Cambridge

Darmann A (2019) Manipulability in a group activity selection problem. Soc Choice Welf 52:527557

Hardin G (1968) The tragedy of the commons. Science 162:1243-1248

Jackson MO, Nicolò A (2004) The strategy-proof provision of public goods under congestion and crowding preferences. J Econ Theory 115:278-308

Long Y (2019) Strategy-proof group selection under single-peaked preferences over group size. Econ Theory 68:579-608

Massó J, Nicolò A (2008) Efficient and stable collective choices under gregarious preferences. Games Econ Behav 164:591-611

Tiebout CM (1956) A pure theory of local expenditures. J Political Econ 64:416-4244 